\title{
Identifying a ten-microRNA signature as a superior prognosis biomarker in colon adenocarcinoma
}

\author{
Rong Ma ${ }^{1,2}$, Yanyun Zhao ${ }^{1,2}$, Miao He $e^{1,2}$, Hongliang Zhao ${ }^{1,2}$, Yifan Zhang ${ }^{1,2}$, Shuqi Zhou ${ }^{1,2}$, Mengcong Gao ${ }^{1,2}$,
} Di Di ${ }^{1,2}$, Jue Wang ${ }^{1,2}$, Jian Ding ${ }^{1,3^{*}}$ and Minjie Wei ${ }^{1,2^{*}}$ (D)

\begin{abstract}
Background: Increasing studies have suggested that aberrant expression of microRNAs might play essential roles in the progression of cancers. In this study, we sought to construct a high-specific and superior microRNAs signature to improve the survival prediction of colon adenocarcinoma (COAD) patients.

Methods: The genome-wide miRNAs, mRNA and IncRNA expression profiles and corresponding clinical information of COAD were collected from the TCGA database. Differential expression analysis, Kaplan-Meier curve and timedependent ROC curve were calculated and performed using R software and GraphPad Prism7. Univariate and multivariate Cox analysis was performed to evaluate the prognostic ability of signature. Functional enrichment analysis was analyzed using STRING database.

Results: We identified ten prognosis-related microRNAs, including seven risky factors (hsa-miR-197, hsa-miR-32, hsa-miR-887, hsa-miR-3199-2, hsa-miR-4999, hsa-miR-561, hsa-miR-210) and three protective factors (hsa-miR-3917, hsa-miR-3189, hsa-miR-6854). The Kaplan-Meier survival analysis showed that the patients with high risk score had shorter overall survival (OS) in test series. And the similar results were observed in both validation and entire series. The time-dependent ROC curve suggested this signature have high accuracy of OS for COAD. The Multivariate Cox regression analysis and stratification analysis suggested that the ten-microRNA signature was an independent factor after being adjusted with other clinical characteristics. In addition, we also found microRNA signature have higher AUC than other signature. Furthermore, we identified some miRNA-target genes that affect lymphatic metastasis and invasion of COAD patients.
\end{abstract}

Conclusion: In this study, we established a ten-microRNA signature as a potentially reliable and independent biomarker for survival prediction of COAD patients.

Keywords: Colon adenocarcinoma, MicroRNAs, Biomarker, Prognosis

\footnotetext{
*Correspondence: jding@simm.ac.cn; weiminjiecmu@163.com

1 Department of Pharmacology, School of Pharmacy, China

Medical University, No.77 Puhe Road, Shenyang North New Area, Shenyang 110122, Liaoning, China

Full list of author information is available at the end of the article
}

\section{$\triangle B M C$}

a adaptation, distribution and reproduction in any medium or format, as long as you give appropriate credit to the original author(s) and the source, provide a link to the Creative Commons licence, and indicate if changes were made. The images or other third party material in this article are included in the article's Creative Commons licence, unless indicated otherwise in a credit line to the material. If material is not included in the article's Creative Commons licence and your intended use is not permitted by statutory regulation or exceeds the permitted use, you will need to obtain permission directly from the copyright holder. To view a copy of this licence, visit http://creativeco mmons.org/licenses/by/4.0/. The Creative Commons Public Domain Dedication waiver (http://creativecommons.org/publicdomain/ zero/1.0/) applies to the data made available in this article, unless otherwise stated in a credit line to the data. 


\section{Background}

Colon adenocarcinoma (COAD), the fourth most commonly malignant cancer, has been the fifth leading cause of cancer-related death diseases in worldwide. It was estimated that nearly 101,420 new COAD cases were diagnosed and 27,640 deaths in the United States in 2019 [1]. Despite diagnostic methods and comprehensive treatment have been developed during the past few years, the overall 5 -year survival rate of COAD patients is still unsatisfactory and the underlying molecular mechanisms of COAD progression are still elusive [2]. Although several biomarkers for COAD have been undergoing or tested in clinical trials, such as carcinoembryofnic antigen (CEA) [3] and so on, many more potential reliable and valuable biomarkers are imperative to be detected and constructed to improve the prognosis of patients with COAD.

MicroRNAs (miRNAs) are a large family of post-transcriptional regulators that are approximately 21 nucleotides in length and control many developmental and cellular processes in eukaryotic organisms [4]. More recently, increasing studies have demonstrated that microRNAs play essential roles in the progression of cancers. Some microRNAs were associated with disease prognosis and clinical outcome, suggesting microRNAs could be one of the best candidates as potential biomarkers for cancers [5, 6].

In addition, compared with a single biomarker, integrating multiple signature model would fundamentally improve the precise of prognostic value [7], and multigene-expression signatures have been reported to predict prognostic in various cancers $[8,9]$. Therefore, searching a panel of microRNA signature might have predictive and prognostic value in patients with COAD.

In the present study, we established an effective microRNAs-based signature to predict the prognosis of COAD taking advantage of TCGA database. And we verified the predictive power of this signature for COAD in validation and entire series. More significantly, we verified our microRNA signature perform better than other signature. Finally, we identified some miRNA-target genes and signaling pathways that promote the progression of COAD patients.

\section{Materials and methods}

\section{COAD patients' information collection}

The microRNAs, mRNA and lncRNA expression profiles of 441 COAD tissues and eight adjacent tissue samples, and their corresponding clinical information were download from The Cancer Genome Atlas of the National Cancer Institute (TCGA, http://cancergenome.nih.gov). The downloaded clinical information summarized in Table 1.
Table 1 Clinical characteristics of COAD in each data set

\begin{tabular}{|c|c|c|c|}
\hline Characteristics & Test series & Validation series & Entire series \\
\hline \multicolumn{4}{|l|}{ Gender } \\
\hline Male & $157 / 300(52.3)$ & $74 / 141(52.5)$ & $231 / 441(52.4)$ \\
\hline Female & $143 / 300(47.7)$ & $67 / 141(47.5)$ & 210/441 (47.6) \\
\hline \multicolumn{4}{|l|}{ Age(years) } \\
\hline$\geq 68$ & $166 / 300(55.3)$ & $71 / 141(50.4)$ & $237 / 441(53.7)$ \\
\hline$<68$ & $134 / 300(44.7)$ & 70/141(49.6) & 204/441 (46.3) \\
\hline \multicolumn{4}{|l|}{ TNM stage } \\
\hline 1 & $55 / 290(19.0)$ & $18 / 140(12.9)$ & $73 / 430(17.0)$ \\
\hline$\|$ & 113/290 (39.0) & $55 / 140(39.3)$ & $168 / 430(39.1)$ \\
\hline III & $82 / 290(28.3)$ & $42 / 140(30.0)$ & $124 / 430(28.8)$ \\
\hline IV & 40/290 (13.8) & 25/140 (17.9) & $65 / 430(15.1)$ \\
\hline \multicolumn{4}{|l|}{ Race } \\
\hline Non-white & 47/191 (24.6) & $24 / 92(26.1)$ & $71 / 283(25.1)$ \\
\hline White & $144 / 191(75.4)$ & 68/92 (73.9) & $212 / 283(74.9)$ \\
\hline \multicolumn{4}{|l|}{ Tstage } \\
\hline $\mathrm{T} 1$ & 8/299 (2.7) & $3 / 141(2.1)$ & $11 / 440(2.5)$ \\
\hline $\mathrm{T} 2$ & $55 / 299(18.4)$ & 20/141 (14.3) & $74 / 440(16.8)$ \\
\hline T3 & 200/299 (66.9) & 100/141 (70.9) & $300 / 440(68.2)$ \\
\hline T4 & $36 / 299(12.0)$ & 18/141 (12.8) & $55 / 440(12.5)$ \\
\hline \multicolumn{4}{|l|}{ N stage } \\
\hline No & $179 / 300(59.7)$ & 78/141 (55.3) & $257 / 441(58.3)$ \\
\hline N1 & $70 / 300(23.3)$ & $34 / 141(24.1)$ & $104 / 441(23.6)$ \\
\hline N2 & $51 / 300(17.0)$ & 29/141 (20.6) & $80 / 441(18.1)$ \\
\hline \multicolumn{4}{|l|}{ M stage } \\
\hline Mo & 219/259 (84.6) & $101 / 126(80.2)$ & $320 / 385(83.1)$ \\
\hline M1 & $40 / 259(15.4)$ & 25/126 (19.8) & 65/385 (16.9) \\
\hline \multicolumn{4}{|c|}{ Lymphatic invasion } \\
\hline No & $172 / 270(63.7)$ & $71 / 129(55.0)$ & 243/399 (60.9) \\
\hline Yes & $98 / 270(36.3)$ & $58 / 129(45.0)$ & 156/399 (39.1) \\
\hline \multicolumn{4}{|c|}{ Microsatallite instability } \\
\hline No & $58 / 65(89.2)$ & $22 / 26(84.6)$ & 80/91 (87.9) \\
\hline Yes & $7 / 65$ (10.8) & $4 / 26(15.4)$ & $11 / 91(12.1)$ \\
\hline \multicolumn{4}{|c|}{ History colon polyps } \\
\hline NO & 176/261 (67.4) & $76 / 120(63.3)$ & $243 / 372(65.3)$ \\
\hline Yes & 85/261(32.6) & $44 / 120(36.7)$ & $129 / 372(34.7)$ \\
\hline \multicolumn{4}{|l|}{ Cancer status } \\
\hline Tumor free & 139/267 (52.1) & $58 / 125(46.4)$ & 197/392 (50.3) \\
\hline With tumor & $128 / 267(47.9)$ & 67/125 (53.6) & 195/392 (49.7) \\
\hline
\end{tabular}

microRNAs selection and signature building

First, we performed differentially expressed microRNAs analysis between 441 tumor tissues and eight adjacent tissues. Then, the 441 COAD cases in TCGA database were randomly divided into a test series $(\mathrm{N}=300)$ and a validation series $(N=141)$. Next, those differentially expressed microRNAs were analyzed using the univariate and multivariate Cox proportional hazards regression analysis in the test series. Based on the expression and coefficient of microRNAs, an optimal microRNAs 
prognostic signature was established and validated in both the validation and entire series. The microRNAbased risk score formula was constructed as follows:

$$
\text { Risk score }=\sum_{i=1}^{n} \text { Coe } i * \mathrm{EV}_{\mathrm{i}},
$$

In this formula, $\mathrm{n}$ represents the number of microRNAs, Coei indicates the coefficient of every microRNA in the result of multivariate Cox regression analysis, and EVi represents the expression level of the every microRNA.

\section{mRNA and IncRNA signature building}

Differentially expressed mRNA and lncRNA were selected to carry out univariate and multivariate Cox analysis. Then, we established mRNA signature and IncRNA signature based on the expression and coefficient of mRNAs and lncRNAs respectively. The risk score formula as follows:

$$
\text { Risk score }=\sum_{i=1}^{n} \text { Coe } i * \mathrm{EV}_{\mathrm{i}} \text {, }
$$

In this formula, $\mathrm{n}$ represents the number of mRNAs or lncRNAs, Coei indicates the coefficient of every mRNA or IncRNA in the result of multivariate Cox regression analysis, and EVi represents the expression level of the every mRNA or lncRNA.

\section{Target gene prediction and functional enrichment analysis} The target genes of these prognostic miRNAs was predicted using Targetscan (http://www.targetscan.org/) and miRDB (http://www.mirdb.org/). The overlapping target genes in these two databases were considered as miRNA-target genes and used for further analysis. Functional enrichment analysis including cellular component (CC), molecular function (MF), biological process (BP) and Kyoto Encyclopedia of Genes and Genomes (KEGG) pathway analysis were performed by STRING database (https://string-db.org/cgi/input.pl) and visualized using GraphPad Prism7 (GraphPad Software Inc., La Jolla, CA).

\section{Statistical analysis}

Differentially expressed microRNAs, mRNA and lncRNA between the COAD patients and corresponding adjacent tissue samples were calculated by the 'edgeR' package. Volcano plot was calculated and depicted by "volcano" $\mathrm{R}$ package. Venn diagram was drew using online website (http://bioinformatics.psb.ugent.be/webtools/venn/). The OS were analyzed by Kaplan-Meier survival curve analysis and calculated by the log-rank test. The time-dependent receiver operating curve (ROC) was performed to assess the sensitivity and specificity of the signature prognosis prediction.
The Kaplan-Meier survival curve analysis of miRNAtarget genes was performed using ualcan database (http:// ualcan.path.uab.edu/index.html). All $P$ value $(P<0.05)$ was determined statistically significant. All statistical analysis was performed with $\mathrm{R}$ language (https://www.rproject.org/, v3.5.1), SPSS 24.0 software (SPSS Inc., Chicago, IL) and GraphPad Prism7 (GraphPad Software Inc., La Jolla, CA).

\section{Results}

\section{Screening ten microRNAs as potential prognostic markers} for COAD

We identified 358 significantly differentially expressed microRNAs between 443 COAD tissues and 8 adjacent tissues $\left(\left|\log _{2} \mathrm{FC}\right|>1, P<0.05\right)$, including 223 upregulated and 135 downregulated microRNAs. Further, Univariate Cox regression analysis was performed to find out microRNAs related to patients' prognosis in test series $(\mathrm{N}=300)$. The result showed that 29 microRNAs were significantly related to the overall survival of patients with COAD (All $P<0.05$, Table 2). To improve the precise of prognostic effect of microRNAs, those 29 candidate microRNAs were further analysis by multivariate Cox analysis in the test series. Finally, a total of 10 microRNAs were filtered as the candidate factors obviously associated with the prognosis of COAD (All $P<0.05$, Table 3). The differentially expression level of these ten microRNAs in paired COAD patients tissue were shown Additional file 1: Figure S1a, and the microRNAs expression level in 441 COAD patients and eight adjacent normal tissues were shown in Additional file 1: Figure S1b. These results showed that six of these ten microRNAs were significantly higher expression in COAD cancer, and four of them were significantly lower expression in COAD cancer $($ All $P<0.05)$.

\section{Identifying a ten-microRNA signature as potential prognostic indicator of COAD}

To optimize the predictive microRNAs profile, the filtered ten microRNAs were used to construct a predictive microRNAs signature. Based on the expression levels of these ten microRNAs and their coefficient assessed by multivariate Cox analysis, a novel risk score formula was established as follows: Risk $\quad$ score $=(0.5059 *$ hsa-miR-197 $)+(0.3729 *$ hsamiR-32) + $(-0.3905 *$ hsa-miR-3917) $+(0.3766$ *hsamiR-887) + (0.5924*hsa-miR-3199-2) + (0.4152*hsamiR-4999) $+(-0.3412$ *hsa-miR-3189) $+(-0.3851$ *hsamiR-6854) + (0.3264*hsa-miR-561) + (0.2422*hsamiR-210). Among these microRNAs, the coefficients of those seven microRNAs (hsa-miR-197, hsa-miR-32, hsa-miR-887, hsa-miR-3199-2, hsa-miR-4999, hsamiR-561 and hsa-miR-210) were positive, manifesting 
Table 2 Univariable Cox regression analysis to access the prognostic value of each microRNA

\begin{tabular}{llrl}
\hline Gene & HR $^{\mathbf{a}}$ & \multicolumn{1}{l}{$\mathbf{Z}$} & $\boldsymbol{P}_{\text {value }}$ \\
\hline hsa-miR-561 & 1.446513414 & 3.061382338 & 0.002203176 \\
hsa-miR-4999 & 1.455114828 & 2.695463158 & 0.007029085 \\
hsa-miR-197 & 1.724755289 & 2.687503003 & 0.007198846 \\
hsa-miR-3189 & 0.652731227 & -2.624955746 & 0.008666023 \\
hsa-miR-92a-2 & 0.688316473 & -2.608519806 & 0.009093475 \\
hsa-miR-552 & 0.854514732 & -2.599786264 & 0.009328184 \\
hsa-miR-92a-1 & 0.692360881 & -2.590209036 & 0.009591767 \\
hsa-miR-3677 & 0.733293138 & -2.541856222 & 0.011026552 \\
hsa-miR-31 & 1.156774428 & 2.537629414 & 0.01116061 \\
hsa-miR-887 & 1.435971429 & 2.483541477 & 0.013008318 \\
hsa-miR-126 & 1.611809612 & 2.478084312 & 0.013208993 \\
hsa-miR-34b & 1.305240441 & 2.423297739 & 0.015380315 \\
hsa-miR-32 & 1.562669545 & 2.398135451 & 0.01647877 \\
hsa-miR-200a & 0.704267099 & -2.365202179 & 0.01802023 \\
hsa-miR-3199-2 & 1.432538638 & 2.318025641 & 0.020447928 \\
hsa-miR-29b-2 & 1.43193496 & 2.317641614 & 0.020468807 \\
hsa-miR-210 & 1.197112875 & 2.311015882 & 0.020831978 \\
hsa-miR-29b-1 & 1.419044842 & 2.302408969 & 0.02131212 \\
hsa-miR-6854 & 0.728782265 & -2.238559963 & 0.025184562 \\
hsa-miR-328 & 1.460765612 & 2.213610222 & 0.026855604 \\
hsa-miR-153-2 & 1.243537182 & 2.154273514 & 0.031218729 \\
hsa-miR-3917 & 0.771090176 & -2.125123704 & 0.033576292 \\
hsa-miR-576 & 1.38364903 & 2.082214899 & 0.037322845 \\
hsa-miR-149 & 1.278012985 & 2.081591901 & 0.037379762 \\
hsa-miR-323a & 1.338950369 & 2.078569517 & 0.037656935 \\
hsa-miR-1277 & 1.320851357 & 2.054244282 & 0.039952054 \\
hsa-miR-501 & 0.753234657 & -2.009449019 & 0.044489536 \\
hsa-miR-3605 & 1.285862924 & 1.999104141 & 0.045597087 \\
hsa-miR-615 & 1.152363534 & 1.960122196 & 0.04998151 \\
\hline
\end{tabular}

HR Hazard ratio

${ }^{a}$ Values $>1.0$ indicate that expression is positively associated with poor survival

${ }^{\text {b }}$ Likelihood ratio test $P$ value

their COAD driving effect, while the coefficients of other three microRNAs (hsa-miR-3917, hsa-miR-3189 and hsa-miR-6854) were negative, indicating their COAD protecting effect. Based on the prognostic model formula, we calculated and ranked the risk score of each patient. The distribution of risk score, survival status of patients and the expression profiles of ten microRNAs in the test series were shown in Fig. 1a. The results showed that the patients with high risk score have shorter survival time than those with low risk score. The expression levels of seven risky microRNAs were higher in the patients with high risk scores, while the expression levels of three protective microRNAs were higher in the patients with low risk scores. And
Table 3 The HR and $P$ value of each gene

\begin{tabular}{lcll}
\hline Gene & Coefficient $^{\mathbf{a}}$ & HR $^{\mathbf{b}}$ & $\boldsymbol{P}^{\text {value }}$ \\
\hline hsa-miR-3199-2 & 0.5924 & 1.8082 & 0.00081 \\
hsa-miR-4999 & 0.4152 & 1.5146 & 0.00447 \\
hsa-miR-3917 & -0.3905 & 0.6767 & 0.00634 \\
hsa-miR-210 & 0.2422 & 1.274 & 0.00771 \\
hsa-miR-561 & 0.3264 & 1.386 & 0.0091 \\
hsa-miR-6854 & -0.3851 & 0.6804 & 0.01656 \\
hsa-miR-887 & 0.3766 & 1.4574 & 0.01735 \\
hsa-miR-197 & 0.5059 & 1.6585 & 0.02686 \\
hsa-miR-3189 & -0.3412 & 0.7109 & 0.04269 \\
hsa-miR-32 & 0.3729 & 1.4519 & 0.04437 \\
\hline
\end{tabular}

HR Hazard ratio

a Values $>0.0$ and ${ }^{b}$ Values $>1.0$ indicate that expression is positively associated with poor survival

${ }^{c}$ Likelihood ratio test $P$ value

similar results were also observed in both validation $(\mathrm{N}=141)$ and entire series $(\mathrm{N}=441$, Fig. $1 \mathrm{~b}, \mathrm{c})$.

Then, in order to explore the prognostic value of the ten-microRNA signature, a total of 300 patients in test series were separated into high-risk score group $(\mathrm{N}=150)$ and low-risk score group $(\mathrm{N}=150)$. The results showed that the patients in high-risk group have an obviously shorter OS and poor prognosis than the patients in low-risk group $(P<0.0001$, Fig. $2 d)$. And similar results were also observed in both validation $(P=0.00617)$ and entire series $(P<0.0001$, Fig. 1 e, $f)$. Then, the time-dependent ROC curve results from three series showed that the prognostic accuracy of the ten-microRNA signature was $0.822,0.760$ and 0.820 at 1 year; $0.857,0.775$ and 0.838 at 3 year; $0.863,0.797$ and 0.855 at 5 year; $0.845,0.772$ and 0.831 at 7 year, respectively. (Fig. 1g-i). These results suggested that the tenmicroRNA signature had high specificity and sensitivity and could predict the prognosis of patients with COAD effectively. Taken together, this ten-microRNA signature is a meaningful potential biomarker to predict prognosis of COAD patients.

\section{The ten-microRNA signature is a prognostic indicator independent to other clinical characteristics}

To further confirm the predictive effect of the tenmicroRNA signature and other clinical characteristic on the prognosis, the univariate and multivariate Cox analysis were performed in entire series. The result of univariate Cox analysis showed that the ten-microRNA signature and some clinical characteristics (including age, TNM stage, $\mathrm{T}$ stage, $\mathrm{N}$ stage, $\mathrm{M}$ stage, Cancer status and Lymphatic invasion) were obviously correlated with the prognosis of COAD (Fig. 2a and Table 4). 

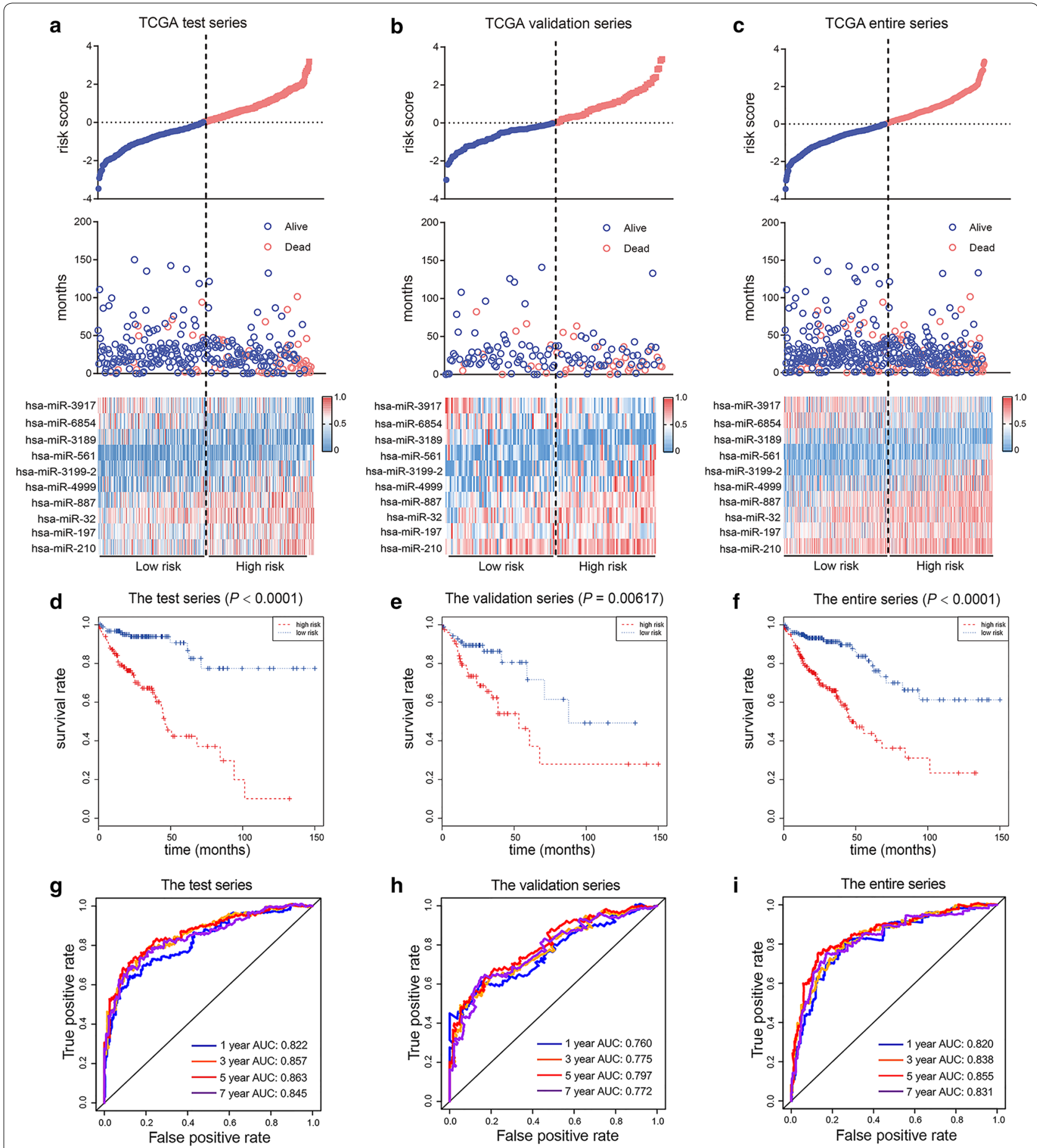

Fig. 1 Identification of ten-microRNA signature associated with prognosis of patients. Risk score distribution, patients'status and heatmap of ten microRNAs expression in test series (a), validation series $(\mathbf{b})$ and entire series $(\mathbf{c})$. Kaplan-Meier analysis of the ten-microRNA signature in test series (d), validation series (e) and entire series ( $\mathbf{f}$. Time-dependent ROC analysis of the sensitivity and specificity of the ten-microRNA signature in test series (g), validation series $(\mathbf{h})$ and entire series (i)

Furthermore, the multivariate Cox analysis showed that the ten-microRNA signature is still an independent prognostic factor $(\mathrm{HR}=2.981,95 \% \mathrm{CI} 1.575-5.642$,
$P=0.001$ ) (Fig. 2b and Table 4). The other conventional clinical characteristics, such as age $(\mathrm{HR}=2.040,95 \% \mathrm{CI}$ $1.132-3.677, P=0.018)$ and Cancer status $(H R=3.126$, 
a

\begin{tabular}{|c|c|c|c|c|c|}
\hline \multirow{2}{*}{ Variables } & \multicolumn{5}{|c|}{ Univariate analysis } \\
\hline & \multicolumn{2}{|r|}{ Hazard Ratio } & HR & $95 \% \mathrm{Cl}$ & $P$ value \\
\hline Risk score & & $\longmapsto$ & 3.684 & $2.359-5.753$ & $<0.0001$ \\
\hline Gender & & $1+$ & 1.132 & $0.764-1.677$ & 0.536 \\
\hline Age & & $\mapsto-1$ & 1.821 & $1.197-2.771$ & 0.005 \\
\hline TNM & & $\longmapsto-1$ & 2.953 & $1.935-4.507$ & $<0.0001$ \\
\hline T stage & & - & 2.759 & $1.275-5.973$ & 0.010 \\
\hline $\mathrm{N}$ stage & & $\longmapsto$ & 3.575 & $2.378-5.373$ & $<0.0001$ \\
\hline M stage & & $\longmapsto$ & 4.294 & $2.736-6.738$ & $<0.0001$ \\
\hline Cancer status & & $\longmapsto$ & 3.330 & $2.009-5.521$ & $<0.0001$ \\
\hline Race & & $y$ & 0.884 & $0.496-1.576$ & 0.676 \\
\hline phatic invasion & & $\longmapsto-1$ & 2.314 & $1.517-3.530$ & $<0.0001$ \\
\hline ry colon polyps & & 들-1 & 0.758 & $0.447-1.284$ & 0.302 \\
\hline & -2 & 0 & & & \\
\hline Lesser ha & & $\rightarrow \mathrm{Gr}$ & & & \\
\hline
\end{tabular}

b

\begin{tabular}{|c|c|c|c|c|}
\hline \multirow{2}{*}{ Variables } & \multicolumn{4}{|c|}{ Multivariate analysis } \\
\hline & Hazard Ratio & HR & $95 \% \mathrm{Cl}$ & $P$ value \\
\hline Risk score & $\longmapsto-1$ & 2.981 & $1.575-5.642$ & 0.001 \\
\hline Age & -5 & 2.040 & $1.132-3.677$ & 0.018 \\
\hline TNM & -1 & 2.081 & $0.949-4.560$ & 0.067 \\
\hline T stage & $\longmapsto$ & 3.180 & $0.737-13.726$ & 0.121 \\
\hline $\mathrm{N}$ stage & H-H & 1.299 & $0.678-2.489$ & 0.431 \\
\hline M stage & -1 & 1.702 & $0.862-3.359$ & 0.126 \\
\hline Cancer status & $\longrightarrow$ & 3.126 & $1.491-6.552$ & 0.003 \\
\hline Lymphatic invasion & 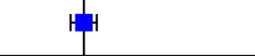 & 0.877 & $0.463-1.663$ & 0.688 \\
\hline & $\begin{array}{l}T \\
5\end{array}$ & & & \\
\hline
\end{tabular}

Fig. 2 Univariate and multivariate Cox analysis of risk score and clinical characteristics. a Univariate Cox analysis of risk score and clinical characteristics. b Multivariate Cox analysis of risk score and clinical characteristics. $P$ value with Bold represents $P<0.05$. Red represents the factor was risky factor and blue represents the factor was protective factor

95\%CI $1.491-6.552, P=0.003)$, were also proven to be independent factors associated with the overall survival (Fig. 2b and Table 4). To further verify prognostic ability of clinical characteristics, we performed Kaplan-Meier analysis. The results were consistent with univariate Cox analysis results and showed that age $(P=0.0116)$, TNM stage $(P<0.0001), \mathrm{T}$ stage $(P=0.0032), \mathrm{N}$ stage $(P<0.0001), M$ stage $(P<0.0001)$, cancer status $(P<0.0001)$ and Lymphatic invasion $(P=0.0004)$ were associated with prognosis of COAD patients (Additional file 2: Figure $\mathrm{S} 2 \mathrm{a}-\mathrm{i}$ ).
Furthermore, stratified analysis were performed to further evaluate whether the ten-microRNA signature exhibit predictive effect within same clinical characteristics. We stratified patients into different group based on age $(<68$ or $>=68)$, TNM stage $(\mathrm{I}+\mathrm{II}$ or $\mathrm{III}+\mathrm{IV}), \mathrm{N}$ stage $(\mathrm{N} 0+\mathrm{N} 1$ or $\mathrm{N} 2), \mathrm{M}$ stage (M0 or M1), Cancer status (tumor free or with tumor) and Lymphatic invasion (yes or no). These results showed that the ten-microRNA signature can still separate patients into high-risk or low-risk group, and patients in high-risk group have shorter OS and poor prognosis than those in low-risk group (Fig. 3a-l). Taken 
Table 4 Univariable and multivariable Cox analysis of the risk score and clinical information in entire series

\begin{tabular}{|c|c|c|c|c|}
\hline \multirow[t]{2}{*}{ Variables } & \multicolumn{2}{|l|}{ Univariate analysis } & \multicolumn{2}{|l|}{ Multivariate analysis } \\
\hline & $\mathrm{HR}^{\mathrm{a}}(95 \% \mathrm{Cl})$ & $P$ value ${ }^{b}$ & $\mathrm{HR}^{\mathrm{a}}(95 \% \mathrm{Cl})$ & $P$ value ${ }^{b}$ \\
\hline \multicolumn{5}{|l|}{ Risk score } \\
\hline Low & 1 (Reference) & & 1 (Reference) & \\
\hline High & $3.684(2.359-5.753)$ & $<0.0001$ & $2.981(1.575-5.642)$ & 0.001 \\
\hline \multicolumn{5}{|l|}{ Gender } \\
\hline Female & 1 (Reference) & & & \\
\hline Male & $1.132(0.764-1.677)$ & 0.536 & & \\
\hline \multicolumn{5}{|l|}{ Age } \\
\hline$<68$ & 1 (Reference) & & 1 (Reference) & \\
\hline$\geq 68$ & $1.821(1.197-2.771)$ & 0.005 & $2.040(1.132-3.677)$ & 0.018 \\
\hline \multicolumn{5}{|l|}{ TNM } \\
\hline$I+\|$ & 1 (Reference) & & 1 (Reference) & \\
\hline$I I I+I V$ & $2.953(1.935-4.507)$ & $<0.0001$ & $2.081(0.949-4.560)$ & 0.067 \\
\hline \multicolumn{5}{|l|}{ Tstage } \\
\hline $\mathrm{T} 0+1$ & 1 (Reference) & & 1 (Reference) & \\
\hline $\mathrm{T} 2+3$ & $2.759(1.275-5.973)$ & 0.010 & $3.180(0.737-13.726)$ & 0.121 \\
\hline \multicolumn{5}{|l|}{ N stage } \\
\hline $\mathrm{NO}+1$ & 1 (Reference) & & 1 (Reference) & \\
\hline N2 & $3.575(2.378-5.373)$ & $<0.0001$ & $1.299(0.678-2.489)$ & 0.431 \\
\hline \multicolumn{5}{|l|}{ M stage } \\
\hline MO & 1 (Reference) & & 1 (Reference) & \\
\hline M1 & $4.294(2.736-6.738)$ & $<0.0001$ & $1.702(0.862-3.359)$ & 0.126 \\
\hline \multicolumn{5}{|l|}{ Cancer status } \\
\hline No & 1 (Reference) & & 1 (Reference) & \\
\hline Yes & $3.330(2.009-5.521)$ & $<0.0001$ & $3.126(1.491-6.552)$ & 0.003 \\
\hline \multicolumn{5}{|l|}{ Race } \\
\hline Non-white & 1 (Reference) & & & \\
\hline White & $0.884(0.496-1.576)$ & 0.676 & & \\
\hline \multicolumn{5}{|c|}{ Lymphatic invasion } \\
\hline No & 1 (Reference) & & 1 (Reference) & \\
\hline Yes & $2.314(1.517-3.530)$ & $<0.0001$ & $0.877(0.463-1.663)$ & 0.688 \\
\hline \multicolumn{5}{|c|}{ Microsatallite instability } \\
\hline No & 1 (Reference) & & & \\
\hline Yes & $26.379(0.127-5479.857)$ & 0.229 & & \\
\hline \multicolumn{5}{|c|}{ History colon polyps } \\
\hline No & 1 (Reference) & & & \\
\hline Yes & $0.758(0.447-1.284)$ & 0.302 & & \\
\hline
\end{tabular}

Italic, significant values $<0.05$

$H R$ hazard ratio; $95 \% \mathrm{Cl}, 95 \%$ confidence interval

a Values $>1.0$ indicate that expression is positively associated with poor survival

${ }^{b}$ Likelihood ratio test $P$ value

together, these results demonstrated that this tenmicroRNA signature was independent risk factors for survival prediction of COAD patients and could stratify patients from different group into subtypes with different prognosis.
The ten-microRNA signature perform better in survival prediction than other signature

To compare the predictive effect between miRNA signature and other signature, we first performed differentially mRNA and lncRNA expression analysis between patients and adjacent tissues. Then, those different 

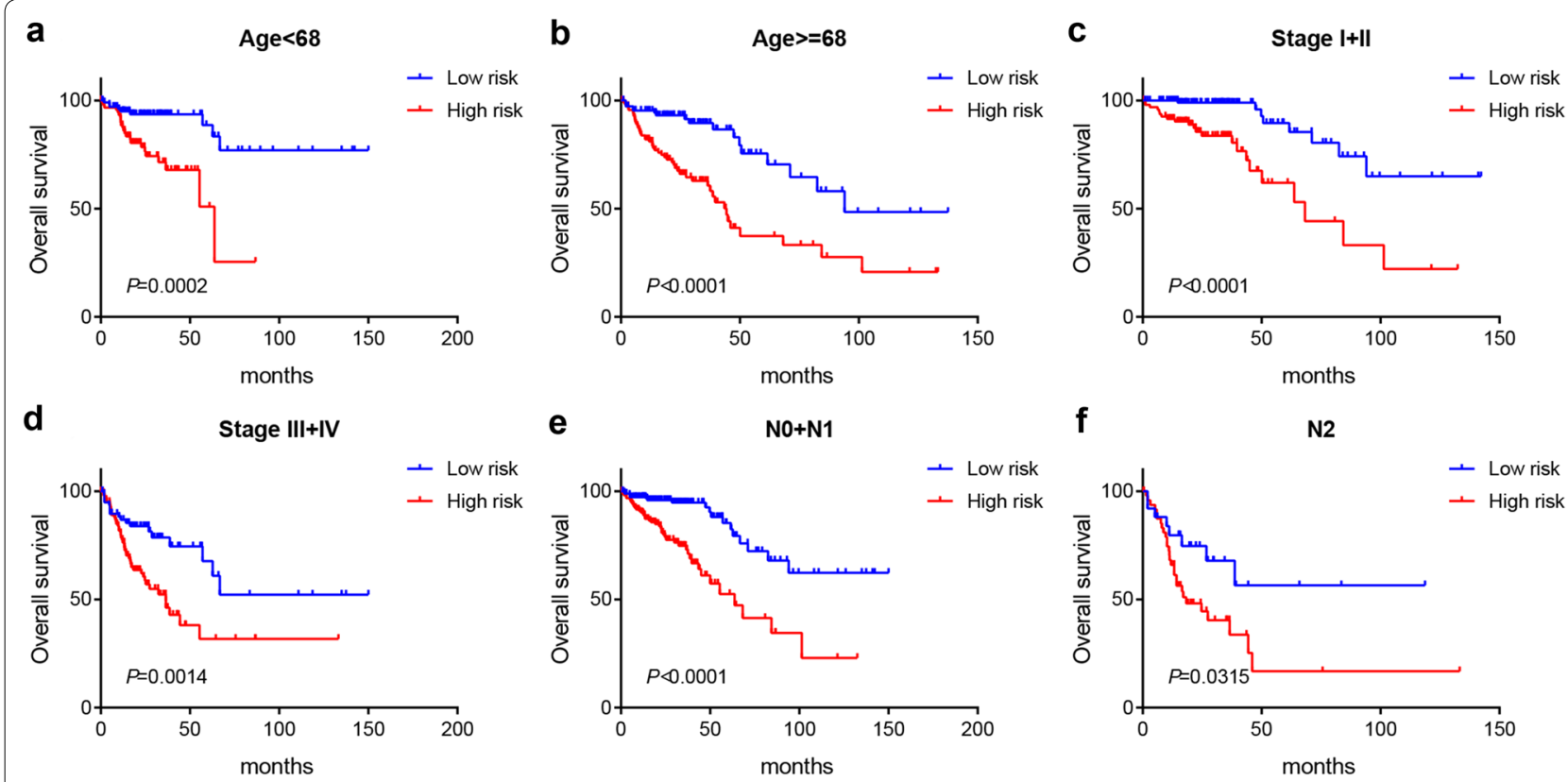

g

Mo

h

M1
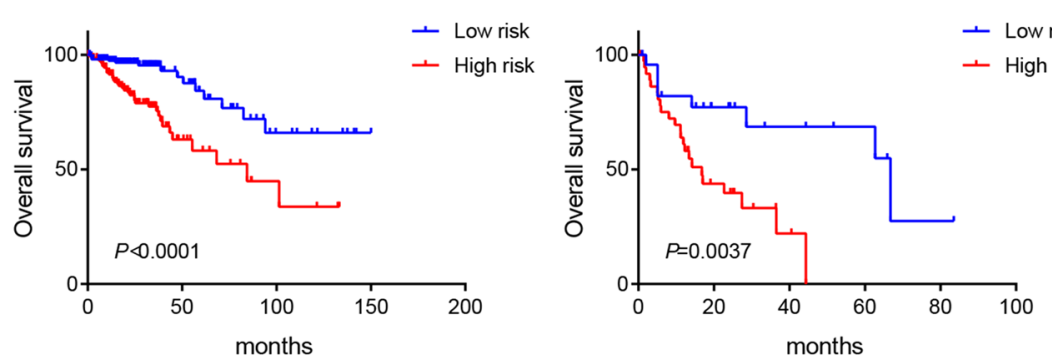

i Cancer status:tumor free
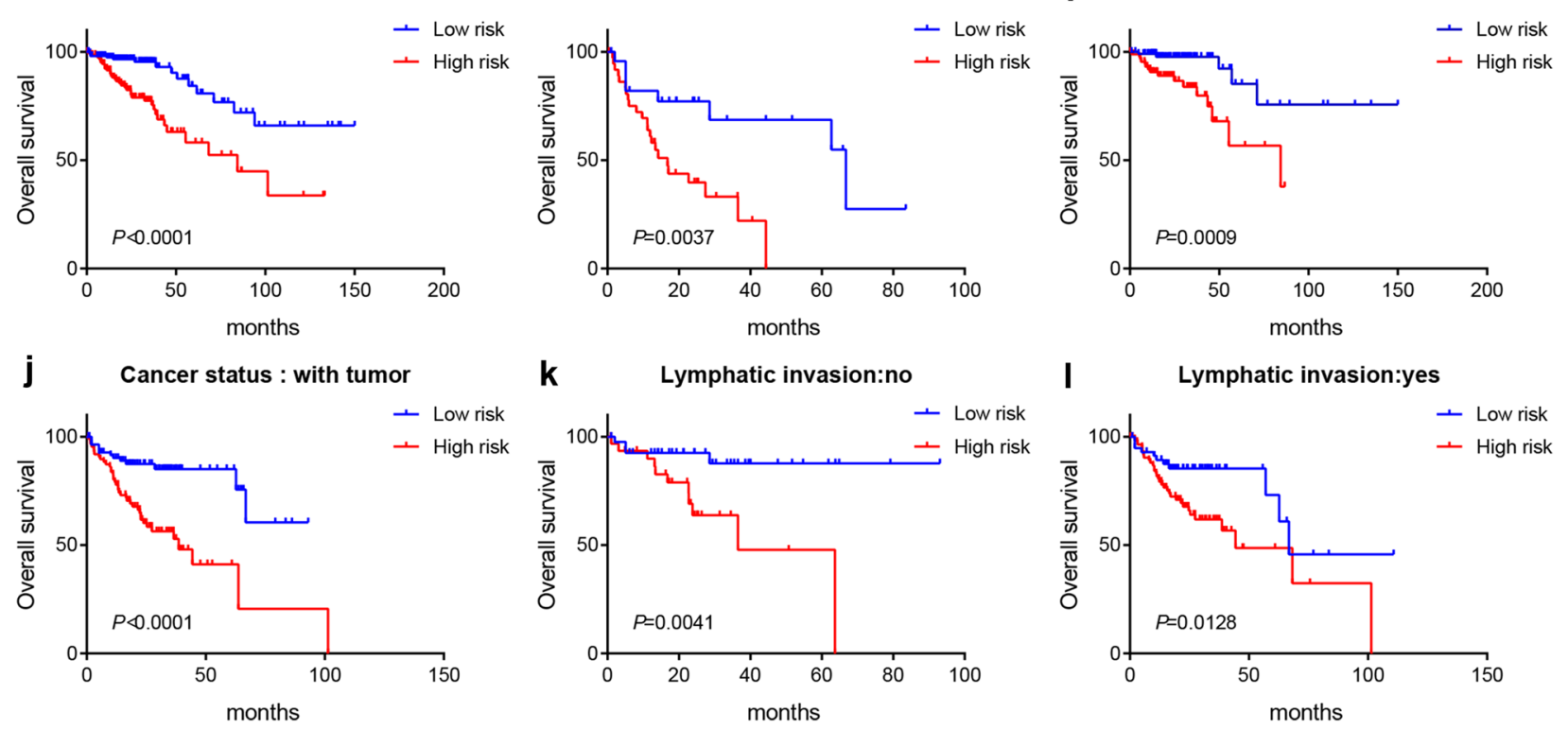

Fig. 3 Stratification analysis of the ten-microRNA signature. Kaplan-Meier analysis of the ten-microRNA signature for patients with different clinical characteristics, including age $<68(\mathbf{a})$ or age $\geq 68(\mathbf{b})$, stage I+ II (c) or stage III + IV (d), N $0+1$ stage $(\mathbf{e})$ or N2 stage (f), M0 stage (g) or M1 stage (h), tumor free (i) or with tumor (j) and no lymphatic invasion (k) or lymphatic invasion (I)

expression mRNA and lncRNA were subjected to univariate and multivariate analysis. Furthermore, we established a mRNA signature and lncRNA signature as follows: $\mathrm{mRNA}$ risk score signature $=\left(-0.2979^{*} \mathrm{CPT} 2\right)$ $+(-0.1522 *$ PPARGC1A $)+(0.2133 * \mathrm{UCN})+(0.0934 * \mathrm{O}$ FCC1 $)+\left(0.1621^{*}\right.$ ATP6V1B1 $)+\left(0.1110^{*}\right.$ LRP2 $)+(0.1052$ *MAGEA1 $)+\left(0.0677^{*}\right.$ HOXC6 $)+(-2.0747 *$ RNU11 $)+$ $(-0.2704 * \mathrm{CD} 1 \mathrm{~B})+\left(0.2944^{*} \mathrm{TYRO} 3 \mathrm{P}\right)$ and lncRNA risk score signature $=\left(0.07464^{*}\right.$ RP11-400N13.2) $+\left(0.12306^{*}\right.$ RP11-742B18.1) + $\left(0.23462^{*}\right.$ RP11-54O7.17) $+\left(0.40607^{*}\right.$ $\mathrm{AC} 002076.10)+\left(0.29414^{*} \quad \mathrm{CTC}-573 \mathrm{~N} 18.1\right)+\left(0.22067^{*}\right.$ $\mathrm{RP} 11-626 \mathrm{H} 12.2)+\left(0.31037^{*} \mathrm{RP} 11-122 \mathrm{~K} 13.7\right)+\left(0.19574^{*}\right.$ RP11-114H21.2). Then, we performed Kaplan-Meier curve analysis (Fig. 4a, b) and time-dependent ROC curve with mRNA signature and lncRNA signature (Fig. 4d, e). These results showed both the mRNA signature and 


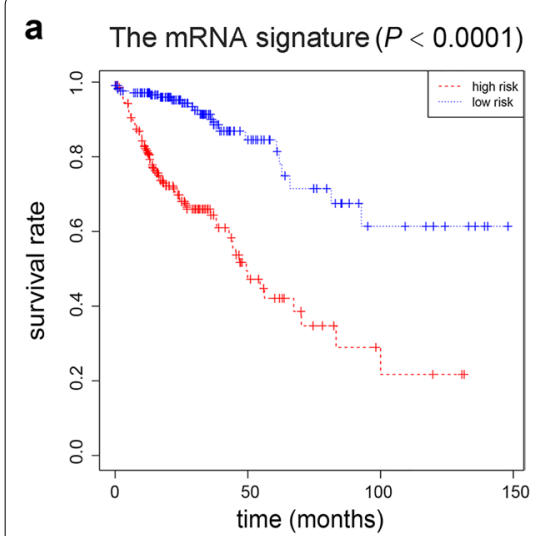

b The IncRNA signature $(P<0.0001)$

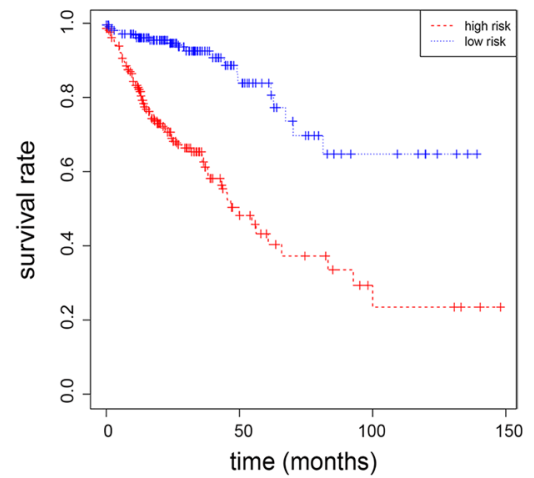

d

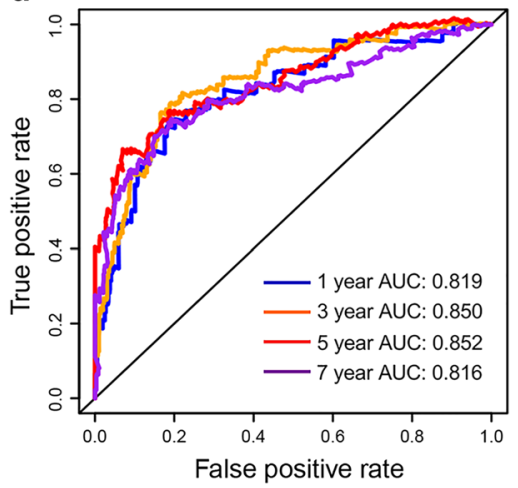

g

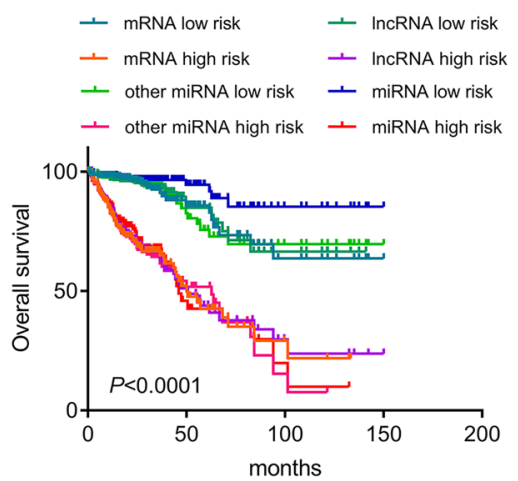

e

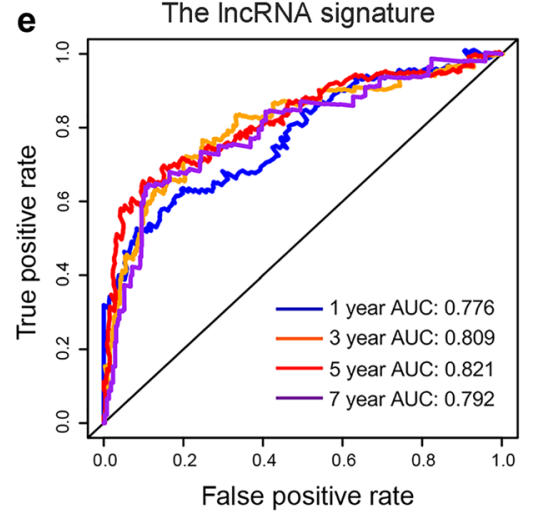

h

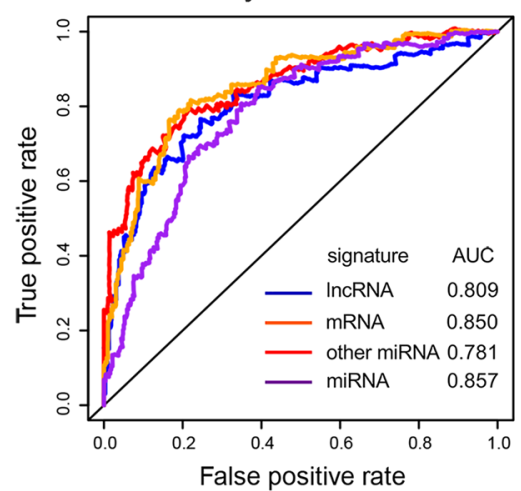

C Other microRNA signature $(P<0.0001)$

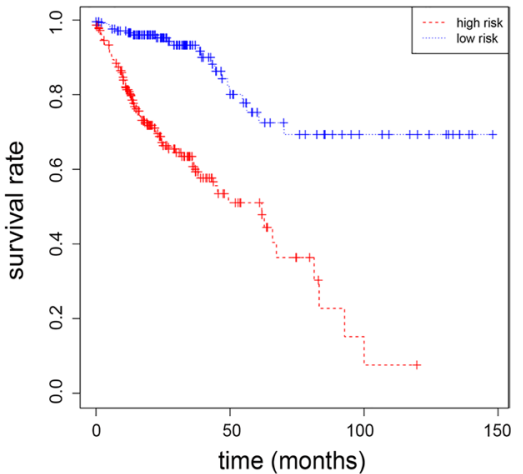

f Other microRNA signature

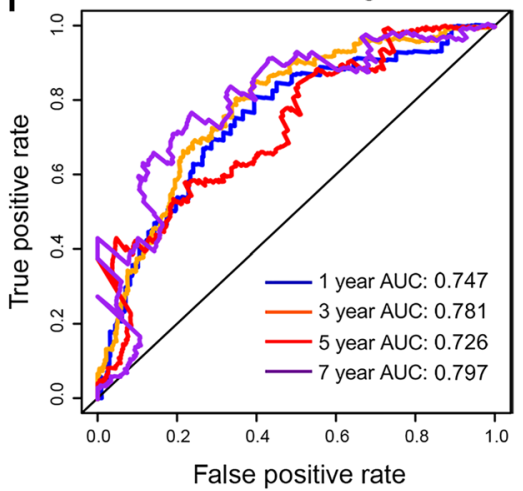

i

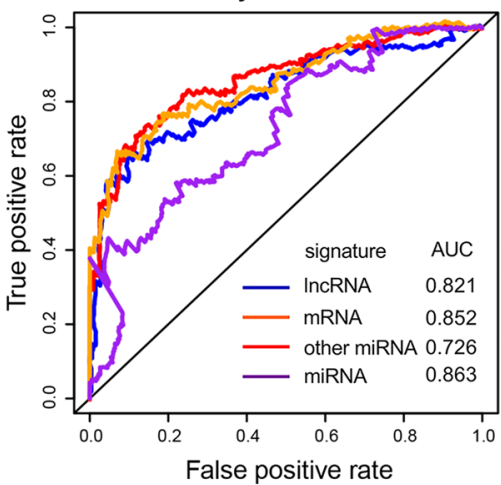

Fig. 4 Comparison of Kaplan-Meier and time-dependent ROC analysis of our microRNA signature with other signature. Kaplan-Meier analysis of the mRNA signature (a), IncRNA signature (b) and other microRNA signature (c). Time-dependent ROC analysis of the sensitivity and specificity of the mRNA signature (d), IncRNA signature (e) and other microRNA signature (f). $\mathbf{g}$ Comparison of Kaplan-Meier analysis of our microRNA signature, mRNA signature, IncRNA signature and other microRNA signature. $\mathbf{h}$ Comparison of 3-year ROC analysis of the sensitivity and specificity of our microRNA signature, mRNA signature, IncRNA signature and other microRNA signature. $\mathbf{i}$ Comparison of 5-year ROC analysis of the sensitivity and specificity of our microRNA signature, mRNA signature, IncRNA signature and other microRNA signature

lncRNA signature exhibited their prognostic value as biomarker for COAD and have high specificity and sensitivity for OS of COAD. In addition, we performed KM plot curve and time-dependent ROC curve with other microRNA signature in previous study (Fig. 4c, f). Next, we compare predictive performance between our microRNA signature and other signature. The Kaplan-Meier curve analysis result showed that patients with our low risk score have significant better survival than those with other low risk score. The time-dependent ROC analysis 
results showed that our microRNA signature have obvious higher AUC than other signature in 3 year and 5 year OS. Taken together, our ten-microRNA signature is a superior indicator for prognosis of COAD patients.

\section{High risk score associates with advanced TNM stage,} lymphatic metastasis and invasion of COAD patients

We further investigated if there was a relationship between the ten-microRNA signature and clinical pathological characteristics. The result showed that TNM stage $(P=0.009), \mathrm{N}$ stage $(P=0.003)$ and Lymphatic invasion $(P=0.001)$ were significantly related to the ten-microRNA signature and patients with high risk score were mainly enriched in stage III + IV, N2 stage and Lymphatic invasion (Table 5). To visualize the relationship between the ten-microRNA signature and other clinical characteristic, we ranked patients according to their risk score, and found obviously asymmetric distribution of the TNM stage, $\mathrm{N}$ stage and Lymphatic invasion (Fig. 5). The result showed that these clinical characteristics, advanced TNM stage (III + IV stage), higher N stage (N2 stage) and Lymphatic invasion were mainly enriched in the higher risk section (Fig. 5a). We further compared the risk score of patients separated by these clinical characteristics. These results showed that the risk score signature was higher in TNM stage III and IV, compared with TNM stage I and II (Fig. 5b). Contrast to the lower $\mathrm{N}$ stage, the risk score signature was higher in N2 stage (Fig. 5c). Further, the risk score signature was mainly higher in Lymphatic invasion (Fig. 5d). However, the other clinical characteristic showed no significant correlation with the ten-microRNA signature (Fig. 5a and Table 5). Taken together, these results indicated that high risk score could predict advanced TNM stage, lymphatic metastasis and invasion of COAD patients.

\section{Incorporation of risk score into $\mathrm{N}$ stage and lymphatic invasion better predicts prognosis of COAD patients}

Since many studies have shown that combined biomarkers are able to improve the prognostic accuracy than a single marker. And our risk score were positively associated with $\mathrm{N}$ stage and lymphatic invasion. Hence, we further investigated whether the incorporation of risk score into $\mathrm{N}$ stage and lymphatic invasion could better predict the prognosis for OS of COAD patients. The time-dependent ROC curve analysis results showed that AUC was $0.879,0.859$ and 0.870 at 3 year; $0.889,0.883$ and 0.892 at 5 year for OS with combined risk score and $\mathrm{N}$ stage, combined risk score and lymphatic invasion and combined risk score, $\mathrm{N}$ stage and lymphatic invasion (Fig. 6). These results demonstrated that combining risk

\begin{tabular}{|c|c|c|c|c|}
\hline \multirow[t]{2}{*}{ Characteristics } & \multirow[t]{2}{*}{$N$} & \multicolumn{3}{|c|}{ Risk score level } \\
\hline & & Low & High & $P$ value ${ }^{a}$ \\
\hline Gender & & & & 0.063 \\
\hline Male & 231 & 125 & 106 & \\
\hline Female & 210 & 95 & 115 & \\
\hline Age (years) & & & & 0.318 \\
\hline$\geq 68$ & 237 & 113 & 124 & \\
\hline$<68$ & 204 & 107 & 97 & \\
\hline TNM stage & & & & 0.009 \\
\hline | and || & 241 & 135 & 106 & \\
\hline III and IV & 189 & 82 & 107 & \\
\hline Tstage & & & & 0.476 \\
\hline $\mathrm{T} 1+\mathrm{T} 2$ & 85 & 45 & 40 & \\
\hline $\mathrm{T} 3+\mathrm{T} 4$ & 355 & 174 & 181 & \\
\hline N stage & & & & 0.003 \\
\hline $\mathrm{NO}+1$ & 361 & 192 & 169 & \\
\hline N2 & 80 & 29 & 51 & \\
\hline M stage & & & & 0.054 \\
\hline MO & 320 & 165 & 155 & \\
\hline M1 & 65 & 25 & 40 & \\
\hline Cancer status & & & & 0.364 \\
\hline Tumor free & 197 & 104 & 93 & \\
\hline With tumor & 195 & 94 & 101 & \\
\hline Race & & & & 0.917 \\
\hline Non-white & 71 & 35 & 36 & \\
\hline White & 212 & 103 & 109 & \\
\hline Lymphatic invasion & & & & 0.001 \\
\hline No & 243 & 137 & 106 & \\
\hline Yes & 156 & 62 & 94 & \\
\hline Microsatallite instability & & & & 0.206 \\
\hline No & 80 & 42 & 38 & \\
\hline Yes & 11 & 8 & 3 & \\
\hline History colon polyps & & & & 0.899 \\
\hline No & 243 & 126 & 117 & \\
\hline Yes & 129 & 66 & 63 & \\
\hline
\end{tabular}

Italic, significant values $<0.05$

a $P$ values were calculated by $X^{2}$ test

score and $\mathrm{N}$ stage and lymphatic invasion could better predict prognosis of COAD patients.

\section{Identifying miRNA-target genes associated with lymphatic metastasis and invasion and poor prognosis of COAD patients}

To explore genes that affect lymphatic metastasis and invasion of COAD patients, we first performed differentially mRNA expression analysis between $\mathrm{N} 0+\mathrm{N} 1$ stage and N2 stage, non-lymphatic invasion and lymphatic 
a

Risk score

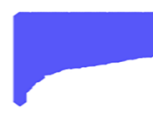

Lymphatic invasion

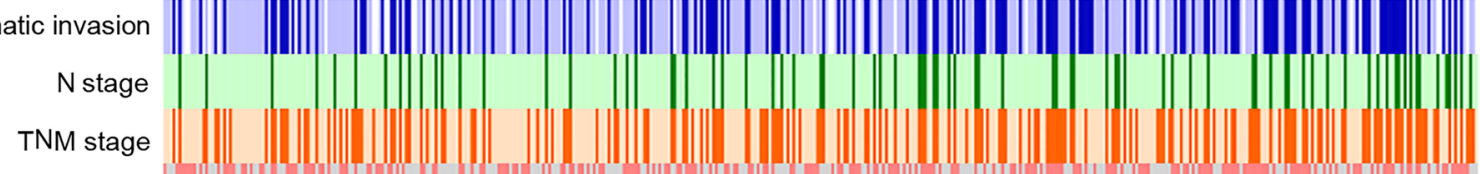

Age

Gender

Tumor

M stage

Cancer status

Microsatallite instability

History colon polyps

Race

Lymphatic

$\mathrm{N}$ stage

TNM stage Age

Age Gender Tumor

M stage

NO

$\begin{array}{ll}\mathrm{N} 0+\mathrm{N} 1 & \mathrm{I} 1 \mathrm{II} \\ \mathrm{N} 2 & \mathrm{III+IV}\end{array}$

Age $<68 \quad$ Female

$\mathrm{T} 1+\mathrm{T} 2 \quad \mathrm{MO}$

Mo
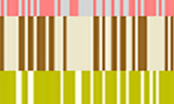

Ires IN2

$\mid I I+I V$

Age $>=68$

IMale

$\mathrm{T} 3+\mathrm{T} 4$

Yes
NO

Microsatallite Race
instability

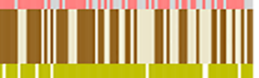

b

TNM stage

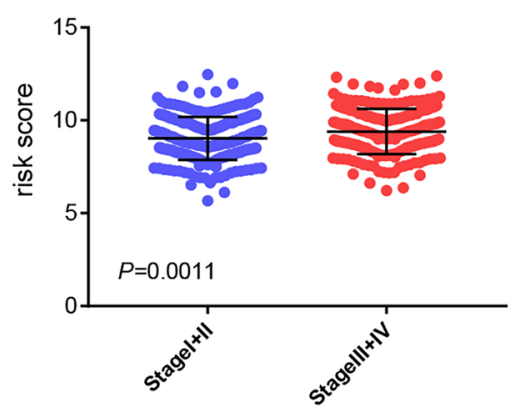

c

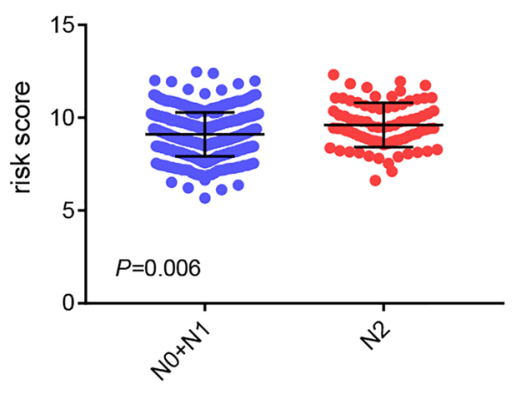

d

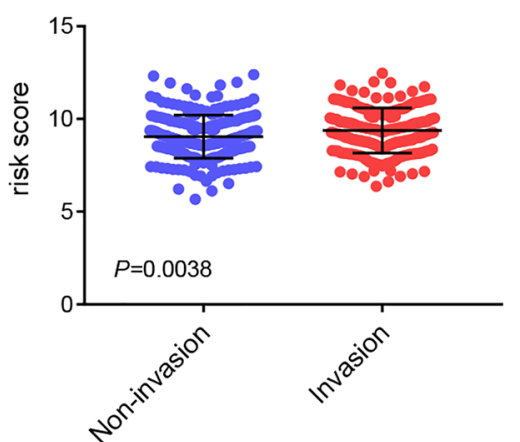

Fig. 5 Relationship between clinical characteristics and the risk score. a The clinical characteristics of patients arranged by the increasing risk score. The distribution of risk score in patients stratified by TNM stage (b), N stage (c) and lymphatic invasion (d)

invasion respectively (Fig. 7a, b). In addition, we also investigated the target genes of the ten microRNAs using two independent miRNA target gene prediction websites: Targetscan and miRDB. Target genes overlapping in the two websites were considered as miRNA-target genes and there are 3248 target genes (Fig. 7c). Then, Venn diagram showed that in these target genes, there are 58 mRNAs were upregulated in N stage, 10 mRNAs were upregulated in lymphatic invasion and 10 mRNAs were upregulated in both $\mathrm{N}$ stage and lymphatic invasion. Furthermore, the Kaplan-Meier curve was performed to analyze the prognostic value of these miRNA-target genes using ualcan website. The results showed that there are $12 \mathrm{mRNAs}$ were associated with prognosis of COAD patients, where KRTAP3-1, SLC35F3 and SLITRK4 were upregulated in both $\mathrm{N}$ stage and lymphatic invasion, while ATP2B2, CILP, ELOVL2, ERBB4, PCDH9, RBM2O, SPTBN4, SYT6 and TMEM132E were upregulated in $\mathrm{N}$ stage. Taken together, these miRNA-target genes might affect the prognosis of COAD patients through promote lymphatic metastasis and invasion of patients. 

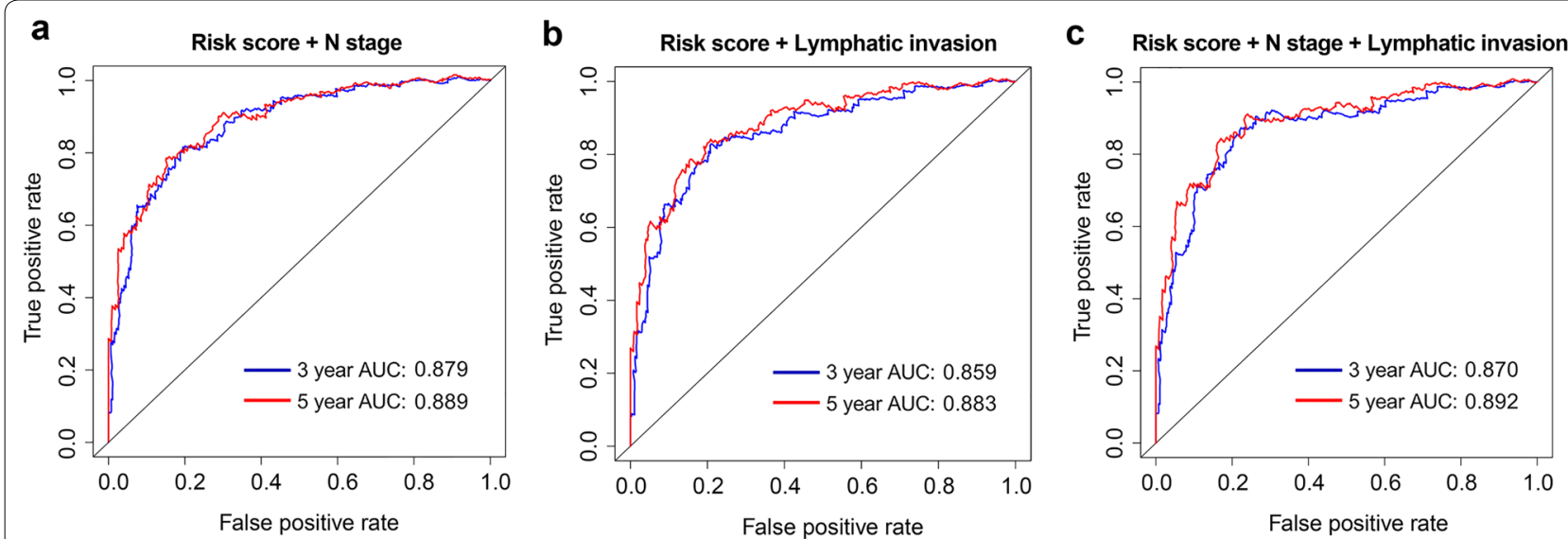

Fig. 6 Time-dependent ROC analysis of combined the risk score and clinical characteristics. a Time-dependent ROC analysis of the sensitivity and specificity of combined the risk score and $\mathrm{N}$ stage. $\mathbf{b}$ Time-dependent ROC analysis of the sensitivity and specificity of combined the risk score and lymphatic invasion. c Time-dependent ROC analysis of the sensitivity and specificity of combined the risk score, $\mathrm{N}$ stage and lymphatic invasion

\section{Identifying biological pathways that the ten-microRNA signature promote lymphatic metastasis and invasion of COAD patients}

We put 78 miRNA-target genes upregulated in $\mathrm{N}$ stage or lymphatic invasion into STRING to explore potentially biological signaling pathways correlated with the tenmicroRNA signature. As shown in Fig. 8, the GO analysis results showed that these target genes were significantly enriched in the Wnt signaling pathway. The KEGG analysis result showed that these target genes were significantly associated with Hippo signaling pathway, MAPK signaling pathway, PI3K-Akt signaling pathway, mTOR signaling pathway, Wnt signaling pathway and so on. Hence, we speculated that these target genes promote progression of COAD patients mainly through Wnt signaling pathway.

\section{Discussion}

COAD is part of colorectal cancer which is a highly heterogeneity of disease with molecular complexity [10]. This feature makes traditional clinical predictive indicators such as stage and so on not enough to predict survival for patients. Although plenty of prognosis biomarkers of COAD has been reported [2, 11], most of them are lacked accuracy and have not been validated in clinical practice. Therefore, novel biomarkers are urgently needed to provide more accurate survival prediction for COAD patients.

During the past years, microRNAs are regarded as novel disease biomarkers because of the universality and stability in cancer [12]. Therefore, in this study, we identified a ten-microRNA signature to better predict the prognosis of COAD patients. Among those selected microRNAs, seven of them (hsa-miR-32, hsa-miR-197,
hsa-miR-210, hsa-miR-3189, hsa-miR-3917, hsamiR-4999, and hsa-miR-6854) were previous reported to have critical roles in colorectal carcinoma [13-16]. Three of them (hsa-miR-3189, hsa-miR-3917 and hsamiR-6854) appear to be protective factors for colon cancer consistent with previous study and four of them (hsa-miR-32, hsa-miR-197, hsa-miR-210 and hsamiR-4999) display to be risky factors for colon cancer also consistent with previous study. The consistency with the reported microRNAs indicated that the selected ways in our study were reasonable. The left three selected microRNAs (hsa-miR-887, hsa-miR-3199-2 and hsamiR-561) have not been identified to be correlated with the prognosis of colon cancer, but these microRNAs have been reported to be correlated with the development, progression, and prognosis of other cancers. For instance, has-miR-887 was reported to be associated with the metastasis and progression of prostate cancer [17]. hasmiR-3199-2 was found to be a prognostic biomarker for papillary renal cell carcinoma [18], and hsa-miR-561 was reported to inhibit gastric cancer cell proliferation and invasion by downregulating c-Myc expression [19]. These results indicated that these microRNAs might also play an important role in colon cancer. In addition, among our screened microRNAs, miR-32, miR-4999, miR-3189, miR-6854, miR-561 and miR-210 were significantly upregulated in COAD patients (Additional File 1: Figure S1a, b), suggesting these microRNAs might exhibit diagnostic value in COAD.

By applying this microRNA signature to the patients, a significantly risk stratification for patients' outcome was observed between survival curves of patients with highrisk or low-risk scores. Patients in the high-risk group have a significantly shorter survival and poor prognosis 


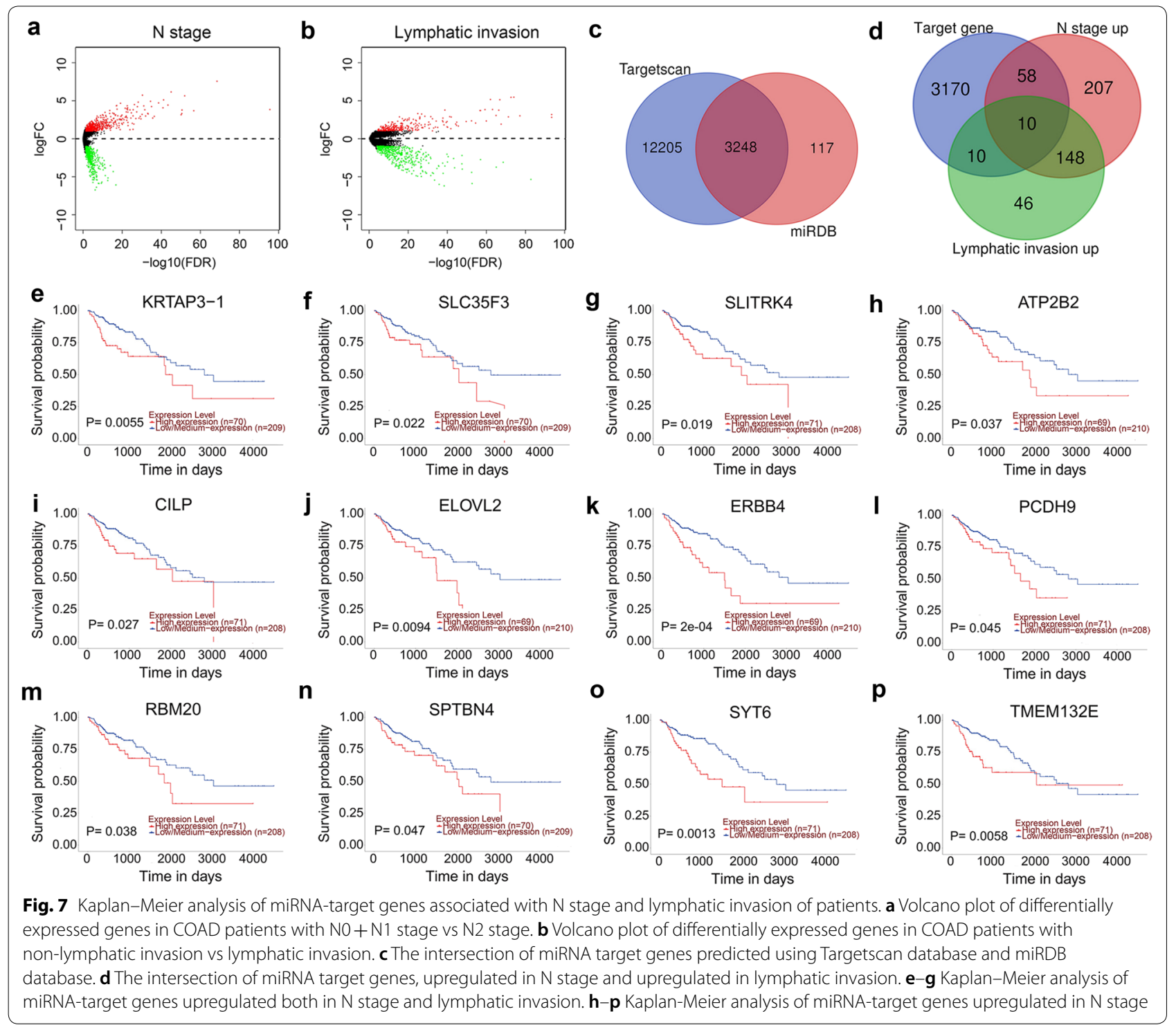

than those in the low-risk group. These results suggested that prognostic value of the microRNA signature is robust and reliable for survival prediction in ccRCC patients. Besides, univariate and multivariate Cox analysis results demonstrated that our microRNA signature is an independent risk factor for prognosis of COAD patients.

At present, although several biomarkers including secretory proteins, mRNA and lncRNA signatures have been used for diagnosis and prognosis in a variety of cancers, they still have themselves limits respectively [2022]. Generally, compared with protein biomarkers, RNA biomarkers are more sensitivity and specificity, whose cost are lower. These results provide a good idea for us to research early diagnosis and prognosis monitoring of patients from the perspective of RNA. Regrettably, mRNA and lncRNA is easily degraded during extraction. However, miRNA as an alternative biomarker have a promising clinical value in diagnosis and prognosis monitoring of patients. More importantly, our microRNA signature have higher accuracy for OS than mRNA, linRNA and other miRNA signature. Therefore, our study provide a novel biomarker with superiority to predict the prognosis of COAD patients.

Tumor metastasis is the chief cause of death in the vast majority of cancer patients including COAD and is indicative for poor prognosis [23, 24]. However, it is strongly correlated with initial tissue invasion at the primary tumor site [25]. Therefore, it is need to point out that novel therapeutic strategies still need to explore to 


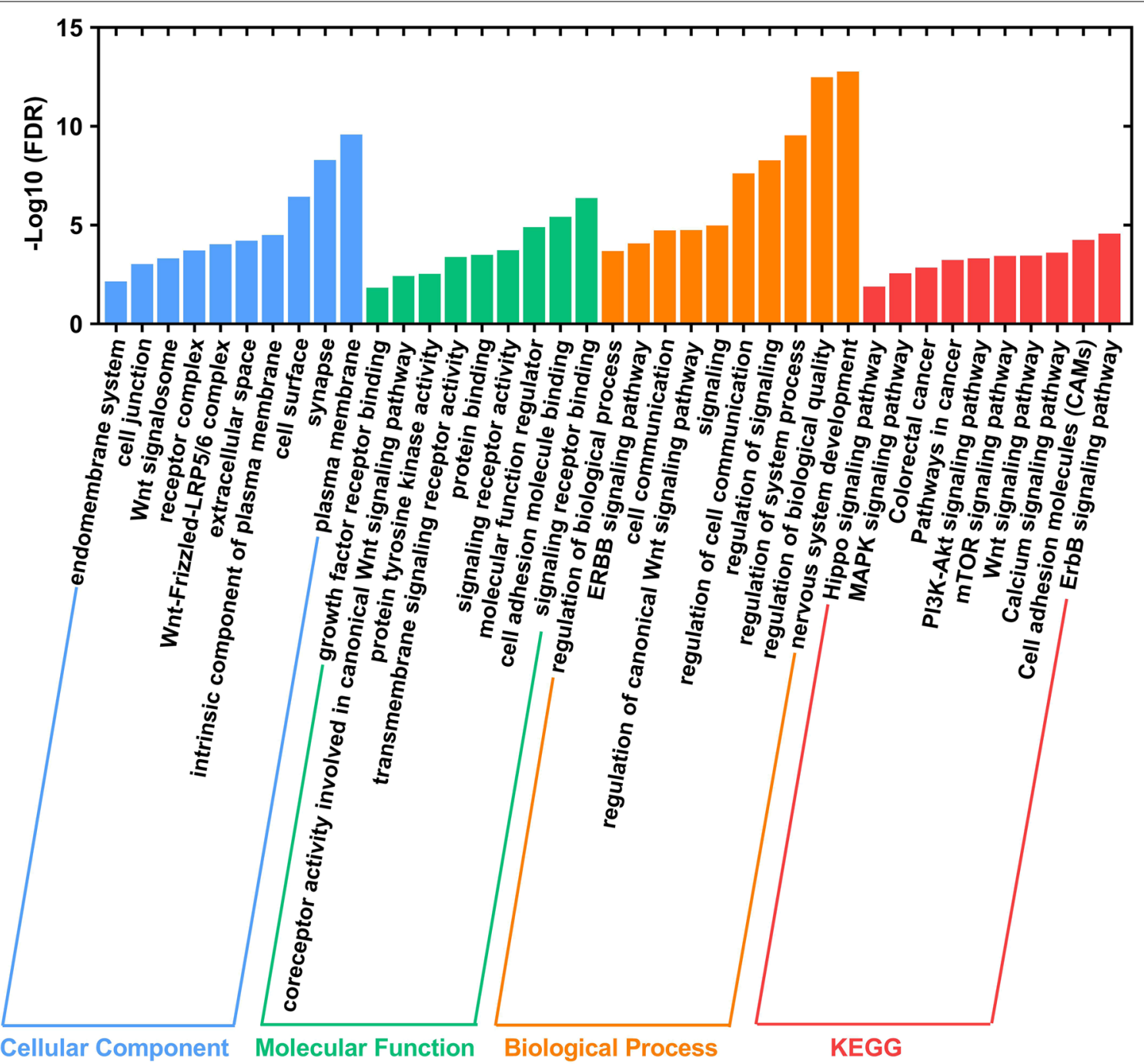

Fig. 8 Functional enrichment analysis of miRNA-target genes upregulated in N stage or lymphatic invasion. Functional enrichment analysis including cellular component, molecular function, biological process and KEGG analysis of 78 miRNA-target genes upregulated in N stage or lymphatic invasion

avoid metastasis. In our study, high risk score indicated advanced TNM stage, higher $\mathrm{N}$ stage and lymphatic invasion, suggesting our signature could affect the progression of COAD patients. Furthermore, we identified some miRNA-target genes that promote lymphatic metastasis and invasion and associate with survival of patients. These target genes might exhibit a helpful indicator for lymphatic metastasis and invasion and prognosis of COAD patients. Among these target genes, ERBB4 has been reported to be associated with colon cancer metastasis, which was consistent with our results [26]. The left of target genes might be novel potential therapeutic targets for patients with lympghatic metastasis and invasion.
Wnt family genes play essential roles in human tumorigenesis. The Wnt signaling pathway could involve in cell proliferation, migration and fate during embryonic development. In adulthood, Wnt signaling pathway exhibit a critical role in regulating homeostasis and self-renewal of tissues. Particularly, in the intestinal epithelium, Wnt signaling pathway promotes proliferation and/or differentiation of stem cells in the intestinal crypts. This is why Wnt signaling pathway play an important role in colon carcinoma [27]. In our study, the GO and KEGG analysis were mainly enriched in Wnt signaling pathway. Hence, we believed that these miRNA-target genes might affect the progression of COAD patients through Wnt signaling pathway. 
In addition, a growing number of studies suggest that combined molecular biomarkers with clinical characteristics are able to improve the prognostic accuracy for patients than a single biomarker. Therefore, we incorporated our signature with $\mathrm{N}$ stage and lymphatic invasion, which resulted in improved predictive accuracy in OS of COAD patients compared to using signature alone. Thus, our microRNA signature could serve as a help indicator to predict prognosis of patients, especially when combined with $\mathrm{N}$ stage and lymphatic invasion.

However, there are also some limitations in this study. First, the prognostic signature of microRNA-based expression were identified by reasonable statistical approaches, but the results was only verified in TCGA database and not verified in clinical practice. Then, although we have some clinical characteristics information in this analysis, several important factors including alcohol consumption, food style, smoking history, and treatment information (surgery, chemotherapy, and radiotherapy) were not available in TCGA database, and we could not control those factors that might cause biases in our analysis. Final, The large scale studies are needed to exam our signature before the ten-microRNA signature can be applied in the clinical practice.

\section{Conclusions}

In this study, we established a ten-microRNA signature as a novel superior and independent indicator to accurately predict prognosis of COAD patients. Furthermore, we identified some miRNA-target genes that affect lymphatic metastasis and invasion and prognosis of COAD patients. These miRNA-target genes were able to be novel therapeutic therapy for patients with lymphatic metastasis and invasion. Finally, we found Wnt signaling pathway might be the significant mechanism in affecting the progression of COAD.

\section{Supplementary information}

Supplementary information accompanies this paper at https://doi. org/10.1186/s12935-019-1074-9.

Additional file 1: Figure S1. The differentially expression of ten microRNAs. a The differentially expression of ten microRNAs in TCGA database between COAD tissues $(N=8)$ and paired adjacent tissues $(N=8)$. b The differentially expression of ten microRNAs in TCGA database between COAD tissues $(\mathrm{N}=441)$ and adjacent tissues $(\mathrm{N}=8)$.

Additional file 2: Figure S2. Kaplan-Meier analysis of clinical characteristics. Kaplan-Meier analysis of age $\mathbf{a}$, gender $\mathbf{b}$, TNM stage $\mathbf{c}$, T stage $\mathbf{d}$, $\mathrm{N}$ stage $\mathbf{e}$, M stage $\mathbf{f}$, cancer status $\mathbf{g}$, lymphatic invasion $\mathbf{h}$, microsatellite instability $\mathbf{i}$.

\section{Abbreviations}

COAD: colon adenocarcinoma; TCGA: The Cancer Genome Atlas; ROC: receiver operating curve; AUC: area under the curve; OS: overall survival.
Acknowledgements

Not applicable.

Authors' contributions

$R M, M J W, J D$ and $Y Y Z$ designed the study. RM, $Y Y Z$ and $M H$ wrote the paper. RM, YYZ, HLZ, YFZ, SQZ, MCG, DD and JW analyzed data. All authors read and approved the final manuscript.

\section{Funding}

This work was supported by National Natural Science Foundation of China and Liaoning joint fund key program (No.U1608281), Double Hundred Program for Shenyang Scientific and Technological Innovation Projects (Z184-020, 100040).

\section{Availability of data and materials}

All data generated or analyzed during this study are included in this published article.

\section{Ethics approval and consent to participate}

Not applicable.

\section{Consent for publication}

Not applicable.

\section{Competing interests}

The authors declare that they have no competing interests.

\section{Author details}

${ }^{1}$ Department of Pharmacology, School of Pharmacy, China Medical University, No.77 Puhe Road, Shenyang North New Area, Shenyang 110122, Liaoning, China. ${ }^{2}$ Liaoning Engineering Technology Research Center, China Medical University, No. 77 Puhe Road, Shenyang North New Area, Shenyang 110122, Liaoning, People's Republic of China. ${ }^{3}$ Division of Anti-tumor Pharmacology, State Key Laboratory of Drug Research, Shanghai Institute of Materia Medica, Chinese Academy of Sciences, Shanghai, China.

Received: 21 August 2019 Accepted: 13 December 2019

Published online: 30 December 2019

References

1. Siegel RL, Miller KD, Jemal A. Cancer statistics, 2019. CA Cancer J Clin. 2019;69(1):7-34.

2. Zhang T, Guo J, Gu J, Wang Z, Wang G, Li H, Wang J. Identifying the key genes and microRNAs in colorectal cancer liver metastasis by bioinformatics analysis and in vitro experiments. Oncol Rep. 2019;41(1):279-91.

3. Amri R, Bordeianou LG, Sylla P, Berger DL. Preoperative carcinoembryonic antigen as an outcome predictor in colon cancer. J Surg Oncol. 2013;108(1):14-8.

4. Bartel DP. MicroRNAs: target recognition and regulatory functions. Cell. 2009;136(2):215-33.

5. Hu Z, Chen X, Zhao Y, Tian T, Jin G, Shu Y, Chen Y, Xu L, Zen K, Zhang C, et al. Serum microRNA signatures identified in a genome-wide serum microRNA expression profiling predict survival of non-small-cell lung cancer. J Clin Oncol. 2010;28(10):1721-6.

6. Zhou H, Tang K, Xiao H, Zeng J, Guan W, Guo X, Xu H, Ye Z. A panel of eight-miRNA signature as a potential biomarker for predicting survival in bladder cancer. J Exp Clin Cancer Res. 2015;34:53.

7. Qu L, Wang ZL, Chen Q, Li YM, He HW, Hsieh JJ, Xue S, Wu ZJ, Liu B, Tang $\mathrm{H}$, et al. Prognostic value of a long non-coding RNA signature in localized clear cell renal cell carcinoma. Eur Urol. 2018;74(6):756-63.

8. Vermeulen J, De Preter K, Naranjo A, Vercruysse L, Van Roy N, Hellemans J, Swerts K, Bravo S, Scaruffi P, Tonini GP, et al. Predicting outcomes for children with neuroblastoma using a multigene-expression signature: a retrospective SIOPEN/COG/GPOH study. Lancet Oncol. 2009;10(7):663-71.

9. Klein EA, Cooperberg MR, Magi-Galluzzi C, Simko JP, Falzarano SM, Maddala T, Chan JM, Li J, Cowan JE, Tsiatis AC, et al. A 17-gene assay to predict prostate cancer aggressiveness in the context of Gleason grade heterogeneity, tumor multifocality, and biopsy undersampling. Eur Urol. 2014;66(3):550-60. 
10. Jones HG, Jenkins G, Williams N, Griffiths P, Chambers P, Beynon J, Harris D. Genetic and epigenetic intra-tumour heterogeneity in colorectal cancer. World J Surg. 2017;41(5):1375-83.

11. Fan $Q$, Liu B. Discovery of a novel six-long non-coding RNA signature predicting survival of colorectal cancer patients. J Cell Biochem. 2018;119(4):3574-85.

12. Hayes J, Peruzzi PP, Lawler S. MicroRNAs in cancer: biomarkers, functions and therapy. Trends Mol Med. 2014;20(8):460-9.

13. Chen E, Li Q, Wang H, Zhang P, Zhao X, Yang F, Yang J. MiR-32 promotes tumorigenesis of colorectal cancer by targeting BMP5. Biomed Pharmacother. 2018;106:1046-51.

14. Zhou N, Sun Z, Li N, Ge Y, Zhou J, Han Q, Zhao L, Bai C. miR197 promotes the invasion and migration of colorectal cancer by targeting insulinlike growth factorbinding protein 3. Oncol Rep. 2018;40(5):2710-21.

15. Pizzini S, Bisognin A, Mandruzzato S, Biasiolo M, Facciolli A, Perilli L, Rossi E, Esposito G, Rugge M, Pilati P, et al. Impact of microRNAs on regulatory networks and pathways in human colorectal carcinogenesis and development of metastasis. BMC Genomics. 2013;14:589.

16. Zhang $H$, Wang Z, Ma R, Wu J, Feng J. MicroRNAs as biomarkers for the progression and prognosis of colon carcinoma. Int J Mol Med. 2018;42(4):2080-8.

17. Stegeman S, Moya L, Selth LA, Spurdle AB, Clements JA, Batra J. A genetic variant of MDM4 influences regulation by multiple microRNAs in prostate cancer. Endocr Relat Cancer. 2015;22(2):265-76.

18. Luo W, Wang L, Luo MH, Huang YZ, Yang H, Zhou Y, Jia HT, Wang XX. hsamir-3199-2 and hsa-mir-1293 as novel prognostic biomarkers of papillary renal cell carcinoma by COX s. J Cell Biochem. 2017;118(10):3488-94.

19. Qian K, Mao B, Zhang W, Chen H. MicroRNA-561 inhibits gastric cancercell proliferation and invasion by downregulating c-Myc expression. Am J Transl Res. 2016;8(9):3802-11.
20. Alpert E, Pinn VW, Isselbacher KJ. Alpha-fetoprotein in a patient with gastric carcinoma metastatic to the liver. N Engl J Med. 1971;285(19):1058-9.

21. Wang Y, Chen L, Wang G, Cheng S, Qian K, Liu X, Wu CL, Xiao Y, Wang X. Fifteen hub genes associated with progression and prognosis of clear cell renal cell carcinoma identified by coexpression analysis. J Cell Physiol. 2019;234(7):10225-37.

22. Zhu X, Tian X, Yu C, Shen C, Yan T, Hong J, Wang Z, Fang JY, Chen H. A long non-coding RNA signature to improve prognosis prediction of gastric cancer. Mol Cancer. 2016;15(1):60

23. Turajlic S, Swanton C. Metastasis as an evolutionary process. Science. 2016:352(6282):169-75.

24. Cao H, Xu E, Liu H, Wan L, Lai M. Epithelial-mesenchymal transition in colorectal cancer metastasis: a system review. Pathol Res Pract. 2015;211(8):557-69.

25. Kramer N, Walzl A, Unger C, Rosner M, Krupitza G, Hengstschlager $\mathrm{M}$, Dolznig $\mathrm{H}$. In vitro cell migration and invasion assays. Mutat Res 2013;752(1):10-24.

26. Chu S, Wang H, Yu M. A putative molecular network associated with colon cancer metastasis constructed from microarray data. World J Surg Oncol. 2017;15(1):115.

27. Sebio A, Kahn M, Lenz HJ. The potential of targeting Wnt/beta-catenin in colon cancer. Expert Opin Ther Targets. 2014;18(6):611-5.

\section{Publisher's Note}

Springer Nature remains neutral with regard to jurisdictional claims in published maps and institutional affiliations.
Ready to submit your research? Choose BMC and benefit from:

- fast, convenient online submission

- thorough peer review by experienced researchers in your field

- rapid publication on acceptance

- support for research data, including large and complex data types

- gold Open Access which fosters wider collaboration and increased citations

- maximum visibility for your research: over $100 \mathrm{M}$ website views per year

At $\mathrm{BMC}$, research is always in progress.

Learn more biomedcentral.com/submissions 